\title{
Local Crystallography: Phases, Symmetries, and Defects from Bottom Up
}

Alex Belianinov, ${ }^{1,2,}$ Qian He, ${ }^{3}$ Mikhail Kravchenko, ${ }^{1,2}$ Stephen Jesse, ${ }^{1,2}$ Albina Borisevich, ${ }^{1,3}$ Sergei V. Kalinin ${ }^{1,2}$

${ }^{1}$ Institute for Functional Imaging of Materials, Oak Ridge National Laboratory, Oak Ridge, TN 37831.

2 The Center for Nanophase Materials Sciences, Oak Ridge National Laboratory, Oak Ridge, TN 37831.

${ }^{3}$ Materials Sciences and Technology Division, Oak Ridge National Laboratory, Oak Ridge, TN 37831.

Advances in high resolution imaging and scanning transmission electron microscopies as well as probe-based microscopies, extend beyond simple visualization of atomic structure of matter by allowing high (10 pm or better) precision measurements of atomic positions. Such a high level of fidelity is sufficient to correlate the length and hence energy of individual bonds, as well as bond angles to functional properties of materials. We introduce an algorithm for local analysis of material structure based on the statistical analysis of individualized, local atomic neighborhoods. Our clustering analysis explores the connectivity of lattice, allowing for efficient chemical description and identification of phases and structural defects in multiphase samples. This analysis lays the framework for building image genomes and structure-property libraries, based on conjoining structural and spectral realms through local atomic behavior.

Algorithms like k-means clustering on multi-phase regions allow identification of minute structural distortions, providing framework for description of local physical functionalities. As a model system, we have chosen mixed oxide Mo-V-M-O (M = Nb, Ta, Te and/or Sb), which is currently the most promising catalyst for many industrially important reactions, such as propane (amm)oxidation.[1] Our algorithm yields absolute positions of each atom in an image, as well as local descriptors such as column intensity and peak width determined at the refinement stage. Significant improvements in atom finding based on target defined search are made over the traditional peak fitting methods. [2, 3] Atom tilts as well as correction approaches based on the recovered image data are explored. [4].The number of nearest neighbors, or the search radius, can be defined separately and are chosen depending on the type analysis. In the simplest case neighbors are chosen based on dominate symmetry, e.g. 6 for hexagonal lattice, or 4/8 for cubic lattice. Once the local neighborhood is established, the clustering algorithm is utilized with an appropriate selection metric of interest to sort the local neighborhoods. Shown in Figure 1(a) is a raw image of Mo-V-M-O mixed MA and M2 phases. We have specified 6 neighbors as well as 6 clusters; the results of a distance to neighbor based metric are shown in Figure 1(b) and a 4 cluster angle to neighbor based metric in Figure 1(c). Rotation is accounted for by always placing the first neighbor atom in the same location relative to the center and filling the rest in a clockwise fashion. The analysis clearly distinguishes different areas of the image based on the similarity of chemical neighborhoods of their constituent atoms, whose role and effects are discussed in detail. Figure 1(d) is a FFT of the overall image shown in Fig. 1(a), panels (e) and (f) show average atomic neighborhoods for 6 and 50 neighbors respectively. Figure 1(g) is a clustering dendrogram for the distance to neighbor case used to define the final number of clusters. We believe this approach paves the way for full information recovery in high resolution imaging as well as allows classification for automatic identification of materials.[5] 

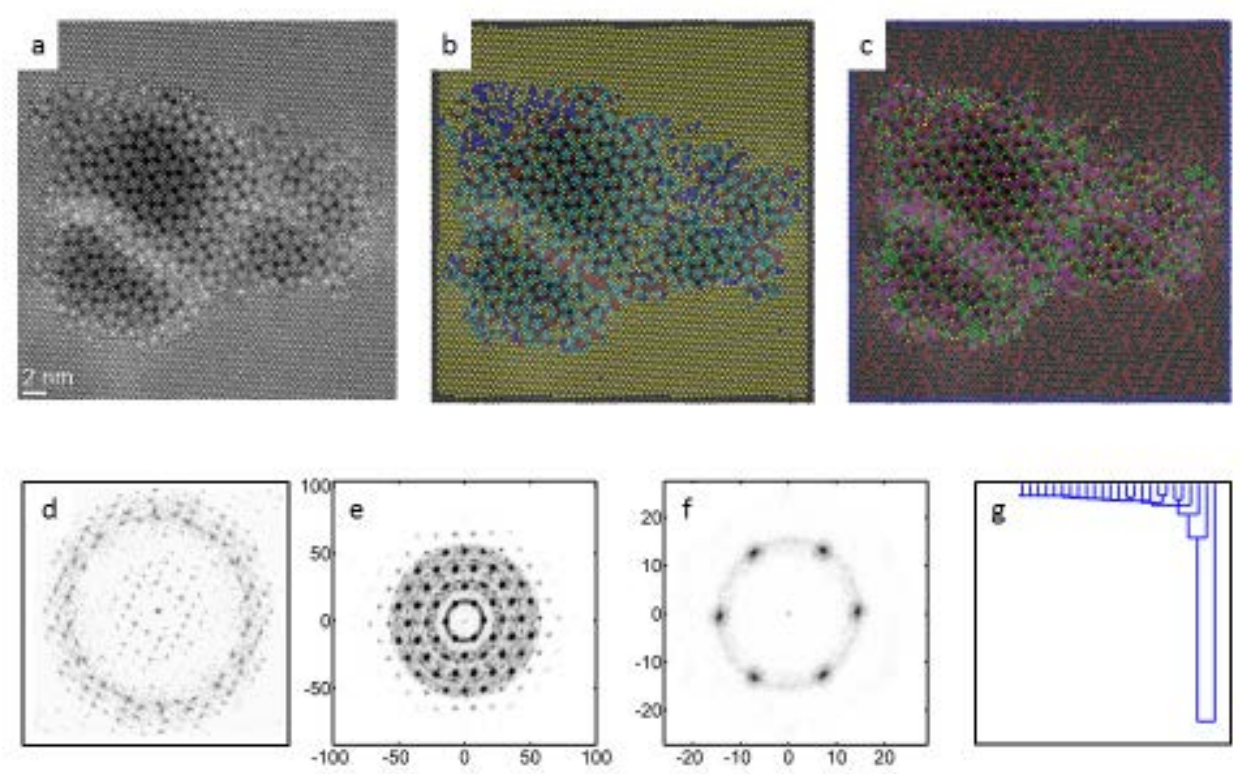

Figure 1. Two phase Mo-V-M-O (M = Nb, Ta, Te and/or Sb). (a) M1 and M2 mixed phase STEM image. (b) k-means clustering results for 6 neighbor, sorted by distance metric. (c) kmeans clustering results for 6 neighbor, sorted by angle metric. (d) FFT of image in (a). (e) 50 member neighborhood of the image in (a). (f) 6 member neighborhood of the image in (a). (g) Dendrogram for the 6 neighbor, sorted by distance metric.

\section{References}

[1] Shiju, N. R. \& Guliants, V. V. Recent developments in catalysis using nanostructured materials. Applied Catalysis A: General 356, 1-17, doi:10.1016/j.apcata.2008.11.034 (2009). [2] Xiahan Sang, Adedapo A. Oni, and James M. LeBeau, Atom Column Indexing: Atomic Resolution Image Analysis Through a Matrix Representation, Microsc. Microanal., doi:10.1017/S1431927614013506

[3] Sarahan M.C., Chi M., Masiel D.J. and Browning N.D; Point defect characterization in HAADF-STEM images using multivariate statistical analysis, Ultramicroscopy, 111 (3) , pp. 251-257; 2011

[4] Jones L., Nellist P.D., Identifying and correcting scan noise and drift in the scanning transmission electron microscope (2013) Microscopy and Microanalysis, 19 (4) , pp. 1050-1060. [5] Research for all authors was supported by the US Department of Energy, Basic Energy Sciences, Materials Sciences and Engineering Division. This research was conducted at the Center for Nanophase Materials Sciences, which is sponsored at Oak Ridge National Laboratory by the Scientific User Facilities Division, Office of Basic Energy Sciences, U. S. Department of Energy. 